\title{
TATTOOING OF CORNEAL OPACITY WITH GOLD AND PLATINUM CHLORIDE
}

\author{
BY
}

\author{
Lt.-Col. Sir Jamshedji N. Duggan, Kt., C.I.E., O.B.E., \\ D.O.(Oxon.), F.C.P.S., A.I.R.O. \\ AND
}

B. P. Nanavati, D.O.M.S., M.R.C.S.(Eng.), L.R.C.P.(Lond.), M.B.B.S.(Bom.)

WHEN a corneal opacity is tattooed, two things are to be borne in mind. The first one is to get a good cosmetic effect and the second one is to obtain a permanent staining of the entire corneal opacity.

A good cosmetic appearance is produced by gold and platinum chloride methods, either of which gives a better appearance, than what is obtained by the old method of tattooing with Indian ink. Since the colour of the iris of Indians is usually dark or light brown, either of the two methods serves the purpose of hiding the white opacity with the stain and matching the colour so obtained with that of the iris.

It is difficult to obtain a permanent staining of the entire area of the leucoma, though soon after the operation it may seem it will be so. Holth ${ }^{1}$ while referring to the tattooing with the platinum chloride solution expresses his opinion that the resulting black colour obtained by this process is more likely to give a permanent black colour than by the gold chloride method. However in the cases which have been tattooed with either gold or platinum chloride and have remained under observation, it has been noticed that the colour has a tendency to fade in a couple of months. But this changed colour has been noticed to remain the same in one case even after five years.

Another drawback is that the stained area rarely remains permanently of the same size. After a few days to a couple of months, it has been observed that the stained area becomes smaller than before on account of the stain having been partly or wholly expelled in places and thus a mottled appearance is produced. In spite of this appearance, the tattooing conceals the leucoma so well that the final cosmetic effect is not at all bad.

Twenty-nine corneal opacities were tattooed with gold chloride and fourteen with platinum chloride. All the operations were performed at Sir C. J. Ophthalmic Hospital except a few which were done in private practice. Out of the twenty-nine cases only five were tattooed with gold chloride and hydrazin hydrate and the 
rest with gold chloride and tannin. All were cases of leucoma with or without adhesion of the iris in the corneal scar. In one case the corneal opacity was produced by the keratitis resulting from a lime burn. In cases where an optical iridectomy was indicated it was usually performed either ten days before or after the tattooing operation. All the cases were operated upon with a view to produce a good cosmetic effect except in one case of aniridia with a central leucoma. In this case an attempt was made to do away with the photophobia by staining a large area of clear cornea besides the central opacity. It was not successful and even the leucoma showed poor staining.

\section{The Operation of Tattooing with Gold Chloride. Instruments and Solutions required}

Speculum, Graefe cataract knife, fixation forceps, cotton applicator, small glass containing a small quantity of gold chloride solution, small pieces of sterile blotting paper, small glass measure containing 1 per cent. tannin solution, if a brown staining be desired or 2 per cent. hydrazin hydrate, if a black colour be required, medicine dropper and normal saline in an undine for washing the eye after using tannin or sterile tap water in case hydrazin hydrate is used.

Technique.-After anaesthetising the eye with 4 per cent. cocaine solution, the speculum is inserted, the eyeball is fixed with fixation forceps at a convenient spot at the limbus and the surface epithelium covering the leucoma and a little beyond it, is scraped off with the knife. The fixation of the eye is given up, the cotton applicator is dipped into the 5 per cent. gold chloride solution and the excess of the fluid is removed by pressing the applicator against the wall of the glass. The eye is fixed again and the applicator is firmly pressed upon the denuded surface for one minute. With a steady patient the fixation of the eyeball can be done away with, three or four such applications each lasting one minute are made. The surface of the eye is continuously kept dry by an assistant with the sterile pieces of blotting paper to prevent irritation of the eye. The tannin or hydrazin solution is dropped on the denuded surface drop by drop for about twenty or thirty seconds until the denuded area becomes stained. Now the eye is washed either with saline or tap water according as the tannin or hydrazin solution has been used. After instilling atropine, vaseline is applied to the eve. Both eyes are bandaged.

Atropine is instilled daily for a week and the dressings changed daily. The unoperated eye is left open on the third day and the operated eye on the ninth day. 
Complications.-None were met with after the operation. Even in cases of adherent leucoma, iritis did not occur. Slight keratitis which resulted from the application of gold chloride always cleared up in a few days.

Comments.-The strength of the gold chloride solution was varied from 2 to 5 per cent. and it was found that 4 or 5 per cent. solutions uniformly gave the best results. A freshly prepared solution was preferable to a stock one which however was serviceable for 6 weeks after which period it was discarded as suggested by Soudakoff ${ }^{2}$. Its strongly acid reaction was made faintly acid to litmus paper with 5 or 10 per cent. sodium bicarbonate solution, to prevent irritation of the operated eye.

Two per cent. hydrazin hydrate solution was always freshly prepared in a small quantity say about 2 drachms. 1 per cent. tannin solution was also freshly prepared before use. Its instillation was more painful than that of hydrazin hydrate which was also preferable to tannin on account of the resulting black colour.

In one case the application of gold chloride was also followed by instillation of adrenalin solution but the staining was very poor. As a reducing agent uviol light was not found so satisfactory as tannin or hydrazin hydrate, though Gifford and Steinberg ${ }^{3}$ obtained good results with it in one case.

The cotton applicator was made by firmly wrapping cotton wool round a wooden stick or a glass rod, and the end of the swab was cut across and trimmed so as to correspond as nearly as possible to the size of the leucoma. It was sterilized with other dressings. In case the surface was found to be too large to be covered by even a large applicator, the area was divided into two segments and each one was treated separately.

A knife and never a trephine was used to prepare the leucoma for impregnation with gold chloride. It was found necessary to scrape the surface right up to the edge of the leucoma to prevent the appearance of a white line around the stained area. The superficial epithelium was always thoroughly removed and it was usually found unnecessary to scarify the leucoma deeply.

A slight keratitis was produced by the gold chloride which came in contact with the cornea surrounding the opacity. However, it cleared in a week or two. The spot at which the eye was fixed with fixation forceps was usually found to be stained slightly. This colour always disappeared in about four weeks. The conjunctiva of the eyeball specially in the lower half got stained, dark grey in colour, which however disappeared in a few days. In a few cases of vascular leucoma it was noticed that the pigment disappeared from a large portion of the tattooed area in a short time. To overcome this difficulty, in one case the blood vessels were divided 
at the limbus as advised by Bartels before thoroughly scraping the surface of the vascular leucoma, but the result was not satisfactory. Some of these cases required to be tattooed a second time.

Those cases, in which the tattooing was not successful at the first attempt, were operated upon a second time after a week; and it was noticed that the second operation which produced a good cosmetic improvement was well borne by the eye in such a short time.

\section{Case Reports}

A few cases are reported below with a view to give an idea of the result of the operation of tattooing with gold chloride.

CASE No. 1.-A Hindu male, aged 25 years, had leucoma of both eyes. Vision of the right eye was $6 / 36$ and that of the left eye fingers at one foot. As desired by the patient only the left eye was operated upon at Sir C. J. Ophthalmic Hospital on August 30,1929 . After scraping the surface of the moderately large leucoma, 5 per cent. solution of gold chloride was applied for 5 minutes and 1 per cent. tannin solution was instilled. The colour changed to dark brown giving a good cosmetic result. He was discharged on September 5, 1929, as he wanted to go to his village.

CASE No. 2.-A Hindu male, aged 30 years, had the adherent leucoma of his left eye tattooed at Sir C. J. Ophthalmic Hospital on the 22nd October, 1929. The opacity was large and vascular. The right eye was normal. 5 per cent. solution of gold chloride was applied for 4 minutes followed by 1 per cent. tannin. The cosmetic effect was good. Optical iridectomy was done on October 29 , 1929, with improvement of vision from a few feet to $6 / 36$. There was very little irritation after either operation. He was discharged on November 3,1929 . When seen 2 years after the operation the pigment had partly faded and disappeared.

CASE No. 3.-A Mahomedan, aged 22 years, had a fairly large leucoma of the right eye. The left eye was normal. An optical iridectomy which was performed on September 10, 1929, at Sir C. J. Ophthalmic Hospital improved his vision from 6/60 to $6 / 24$. On the 17th September, the leucoma was tattooed by applying 5 per cent. gold chloride solution for 4 minutes and instilling 1 per cent tannin solution. Recovery was uneventful and cosmetic improvement good. $\mathrm{He}$ was discharged on September 21, 1929. In this case optical iridectomy was done before tattooing.

CASE No. 4.-A central medium sized vascular leucoma of the right eye of a Hindu male, aged 26 years, was tattooed on 
August 2, 1935, at Sir C. J. Ophthalmic Hospital with 5 per cent. gold chloride solution which was applied for 4 minutes and followed by instillation of 2 per cent. hydrazin hydrate solution. It did not take up the stain well and the few black spots of pigment which were there after the operation disappeared in about 4 days. Hence the process was repeated on August 13, 1935, with good cosmetic result. As there was slight keratitis and some irritation of the eye, he was kept under observation till September 2,1935 , when he left the hospital with a quiet eye showing a black colour.

\section{The Operation of Tattooing with Platinum Chloride, Instruments and Solutions required}

Speculum, fixation forceps, Graefe cataract knife, cotton applicator, sterile pieces of blotting paper, a small glass vessel to hold a small quantity of 2 per cent. platinum chloride solution, a small measure glass containing 2 per cent. solution of hydrazin hydrate, medicine dropper, and sterile tap water in an undine. Both the solutions are freshly prepared before use.

Technique.-The eye is cocainised with 4 per cent. solution of cocaine and the speculum applied. The eyeball is fixed with the fixation forceps at a convenient spot near the limbus whenever required during the operation. The leucoma is scraped with the knife. The solution of platinum chloride is applied with the applicator by firmly pressing it against the denuded surface for one minute. A second similar application is done for another minute. 2 per cent. hydrazin hydrate solution is dropped on the surface of the leucoma for about 25 seconds, during which period the leucoma is seen to turn black. Throughout the operation the excess of solution is removed by an assistant with the sterile pieces of blotting paper. Finally the eye is washed with the sterile tap water, atropine is instilled and vaseline applied before bandaging both the eyes.

Complications.-None were noticed in the cases operated upon except slight keratitis which disappeared in a few days.

Comments.-The 2 per cent. strength of platinum chloride was always found to be suitable for tattooing. Stock solution of platinum and hydrazin hydrate were useless for staining so both of them were always prepared shortly before operation. The time for the application of platinum chloride was always two minutes, but in those cases in which the operation had failed it was increased to three or even four minutes without any harm.

The final colour of the tattooed area was found to change from black to dark grey after about a month. In one case which was 
tattooed five years ago the dark grey colour was found to be the same even after that period. The black colour obtained by tattooing with platinum chloride and hydrazin hydrate was slightly darker in tint than with gold chloride and hydrazin hydrate.

The behaviour of the vascular leucoma with the platinum chloride tattooing was the same as with the gold chloride method. In some cases, the white line surrounding the blackened leucoma, on close inspection looked more prominent with the platinum chloride tattooing than with the gold chloride and tannin because of the contrast in colours.

\section{Case Reports}

There was nothing unusual about the fourteen cases tattooed with platinum chloride with the exception of two which are briefly described below.

CASE No. 1.-A Mahomedan, aged 32 years, wanted to have the adherent leucoma of his left eye tattooed. An optical iridectomy had previously been done and the vision of that eye was $6 / 18$, while that of the right eye was $6 / 6$. The leucoma which was fairly large was tattooed at the Sir C. J. Ophthalmic Hospital on November 15, 1929, with platinum chloride, but it showed no staining of its white surface after completion of the operation. The tattooing with 2 per cent. platinum chloride and hydrazin hydrate solution which was repeated the next day, resulted in black staining of the entire leucoma. No complications occurred, and the eye quieted down. A thin white line surrounded the blackened area all round and looked rather unsightly on close inspection. He was discharged on November 25, 1929. On December 28, 1929, he was again examined as an out-patient, when the black colour was noticed to have slightly faded. When he was seen again on February 3, 1930, the colour had not faded further but the stain had been expelled from a small portion of the leucoma on the inner side. The white line was still there.

CASE No. 2.-A Parsi male, aged 23 years, wanted to have the corneal opacity of his left eye tattooed. It was a fairly large opacity which had resulted from a lime burn of the conjunctiva. An iridectomy was first performed on August 15, 1930. The vision of the left eye had improved to $6 / 60$ from finger counting at about 3 feet, while that of the right eye was already 6/6. After a month the tattooing of the left eye with 5 per cent. gold chloride and 1 per cent. tannin solution was done. The time of application was 4 minutes. It stained the opacity poorly and in a fortnight very few particles of pigment could be seen. The eye which was slightly irritable quieted down in about 15 days. 
Another tattooing with application of 2 per cent. platinum chloride for 2 minutes and instillation of 2 per cent. hydrazin hydrate was done on October 3, 1930. The opacity was fairly well stained and was jet black in colour. However in about a month the colour faded a little and the stain had partly come away producing a mottled appearance which is the same even now after five years, and the cosmetic effect also fairly good.

\section{Summary}

1. Twenty-five cases were tattooed with gold chloride and tannin and four with gold chloride and hydrazin hydrate with fairly good cosmetic result. The use of tannin which produced a brown tint was more painful than hydrazin hydrate which gave a black colour.

2. A slightly acid 4 or 5 per cent. solution of gold chloride was applied to the denuded surface of the leucoma for 4 or 5 minutes. The strength of tannin was 1 per cent. while that of hydrazin hydrate 2 per cent.

3. In vascular leucoma the colour obtained by tattooing with either method slightly faded and partly disappeared in some cases which required a second operation.

4. Fourteen cases were tattooed with freshly prepared 2 per cent. solution of platinum chloride and 2 per cent. solution of hydrazin hydrate. The colour obtained by this method was iet black.

5. Iridectomy could be done either before or after tattooing with either method.

6. Out of the cases reported in this article two are of special interest. One which was tattooed with gold chloride and tannin showed a fairly good cosmetic effect even after two years, while in the other case tattooed with platinum chloride and hydrazin hydrate, the slightly faded black colour had remained unchanged for five years.

\section{REFERENCES}

1. Holth.-Revival of Galen's corneal staining with iron sulphate and tannic acid must be abandoned. Klin. Monatsbl. f. Augenheilk., Vol. LXXXV, August, 1930; p. 806, December, 1930.

2. Sondakoff.-Further observation on tattooing the cornea with gold chloride method (Knapp's method). Nat. Med. Jl., China, Vol. XVII, p. 75, February, 1931.

3. Gifford, Sanford R. and Steinberg, A.-Gold and silver impregnation of cornea for cosmetic purposes. Amer. Jl. of Ophthal., p. 241, April, 1927. 